\title{
NGC 6822: short period variable stars, stellar population and distance scale
}

\author{
Lara Baldacci \\ Bologna University, via Ranzani 1, I-40127 Bologna
}

Luca Rizzi

INAF - Padova Observatory, vicolo dell'Osservatorio 5 I-35122 Padova

Gisella Clementini

INAF - Bologna Observatory, via Ranzani 1 I-40127 Bologna

Enrico V. Held

INAF - Padova Observatory, vicolo dell'Osservatorio 5 I-35122 Padova

\begin{abstract}
Results are presented on a study of the short period variable stars in the dwarf irregular galaxy NGC 6822. We observed an almost uniformly populated classical instability strip from the Horizontal Branch up to the Classical Cepheid region. The main goal we achieved from the analysis of the faint sample is the first detection of RR Lyrae stars in this galaxy.
\end{abstract}

\section{Introduction}

The aim of the present project is the study of the dwarf irregular (dI) galaxy NGC 6822 using its pulsating variable star content. Pulsating variables are tracers of different stellar population in galaxies and are useful for testing theoretical models. Moreover, some of them, such as the RR Lyrae stars and the Classical Cepheids, are primary distance indicators in the Local Group.

\section{Observation and analysis}

Time-series imaging of NGC 6822 was obtained at the VLT (36 V and $11 \mathrm{~B}$ images) in 2001 August. Photometric reductions were performed using DAOPHOT and ALLFRAME (Stetson 1994) and the candidate variables were identified using ISIS2.1 (Alard 2000). About 450 bona fide candidate variables were detected, and all are being analysed with GRATIS (GRaphycal Analyzer of TIme Series; see Clementini et al. 2000) in order to check their actual variation, to classify them and to determine their pulsational characteristics. 


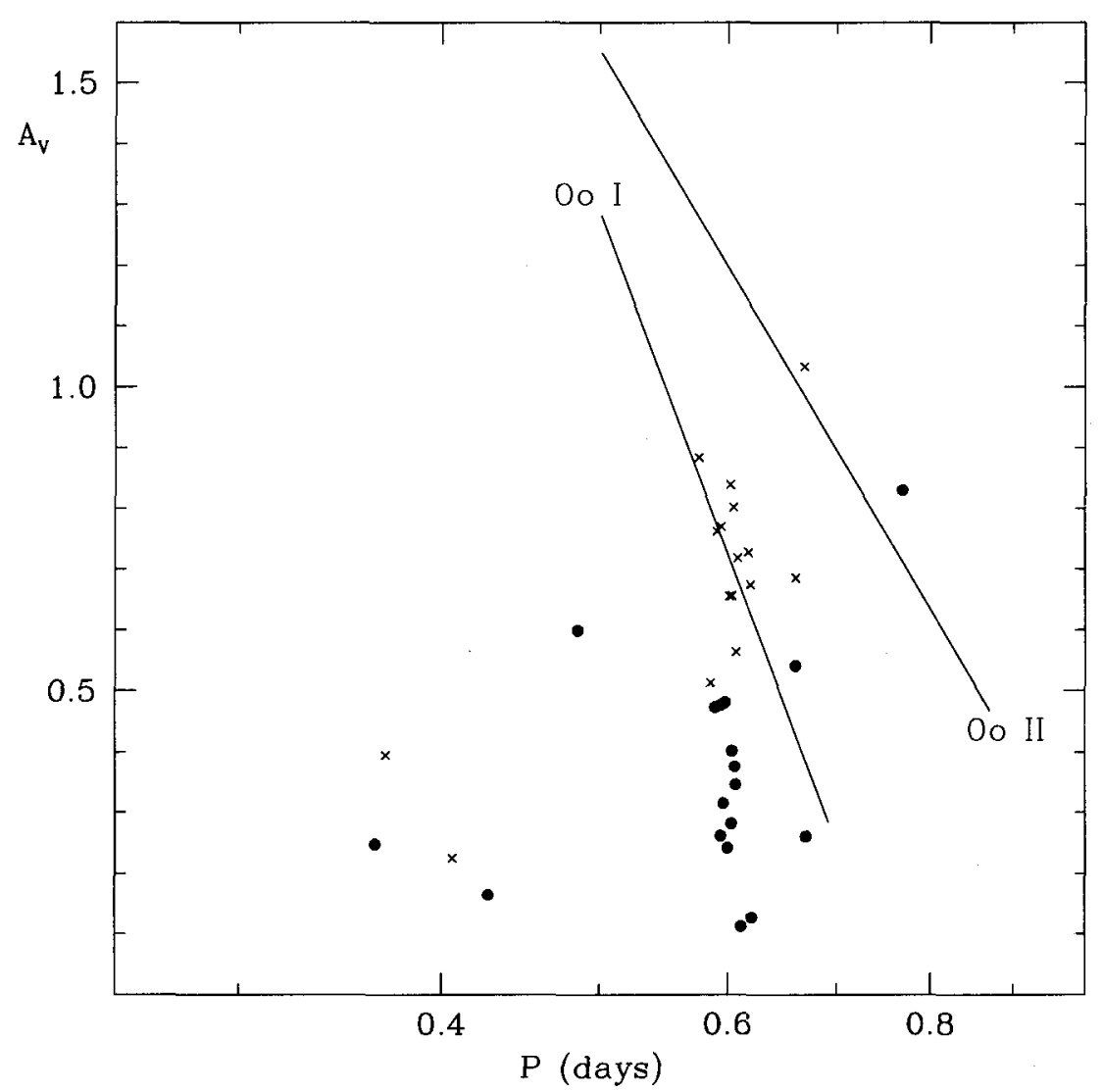

Figure 1. P/A relation for RR Lyr stars (crosses) and low luminosity (LL) Cepheids (dots). Lines show the P/A relations for Oosterhoff type I and II Galactic globular cluster types (Clement \& Rowe 2000).

\section{Result}

The color magnitude diagram of NGC 6822 shows a significant population of candidate variable stars of different types that populate all the regions where the theoretical models predict their presence (Baldacci et al. 2002). Only the variables with $V \geq 23$ (namely variables in the Horizontal Branch region of the classical instability strip) have been fully analysed so far. Among them we found 17 RR Lyr stars ( 15 ab-type and 2 c-type) and 20 brighter short-period variables that we collectively indicate as low luminosity (LL) Cepheids (Clementini et al. 2003). All these objects have good photometric data and light curve coverage that allow an unambiguous determination of the period. Since the Horizontal Branch of NGC 6822 in completely hidden by the young and intermediate-age star populations, our detection of RR Lyr stars is the first indisputable evidence of the presence of an old stellar population $(t \geq 10 \mathrm{Gyr})$ in the galaxy. 


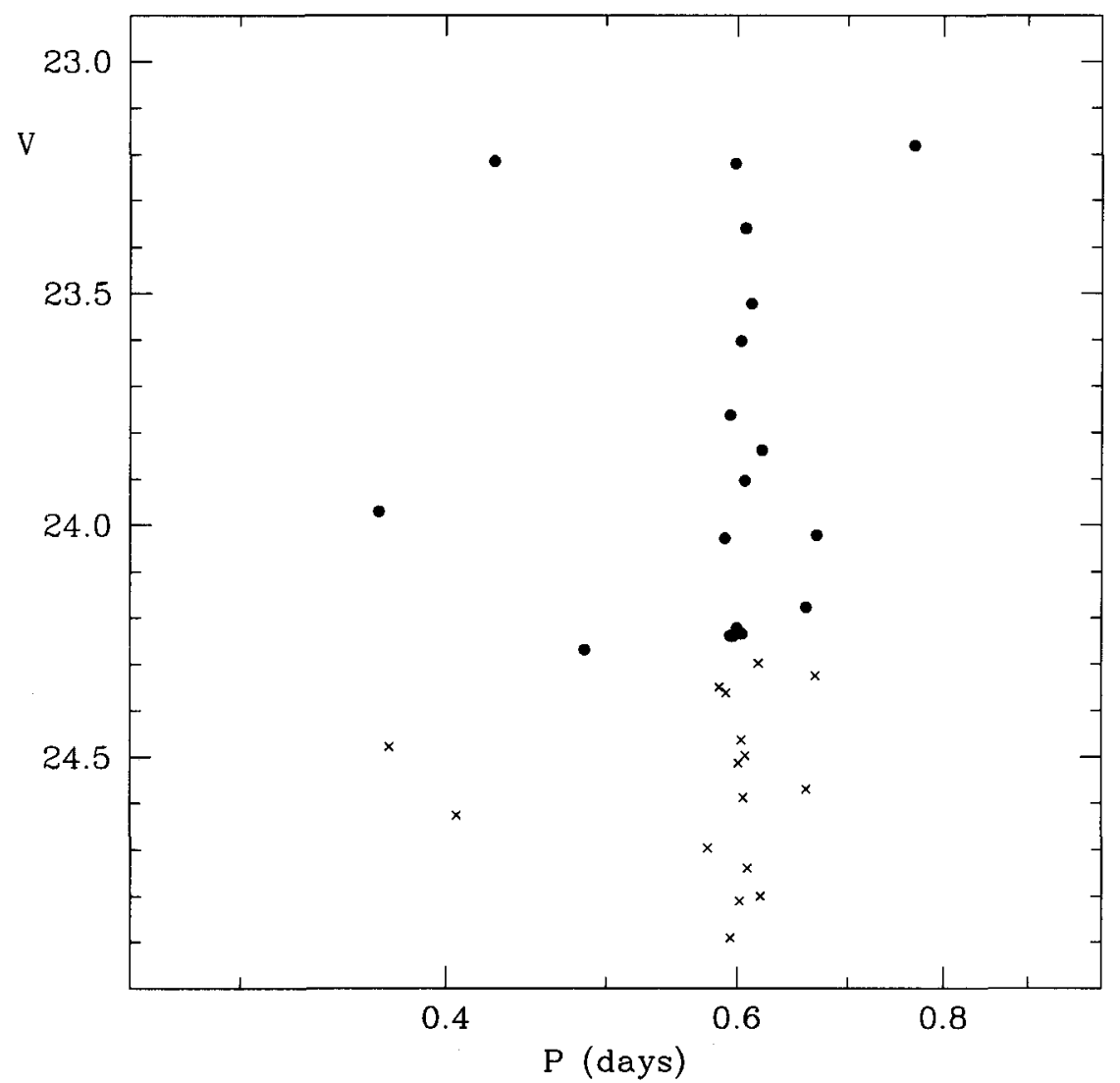

Figure 2. P/L relation for RR Lyrae stars (crosses) and low luminosity (LL) Cepheids (dots).

Figs 1 and 2 show the period-amplitude $(\mathrm{P} / \mathrm{A})$ and the period-luminosity $(\mathrm{P} / \mathrm{L})$ distributions of our sample, respectively. The P/A relation of the RR Lyr stars is similar to the distribution followed by the Galactic globular clusters of Oosterhoff type I (Oosterhoff 1939), although an intermediate distribution (as found in many other dwarf galaxies) is not definitely ruled out by our data. The LL Cepheids are characterized by smaller amplitudes (at a given period) than the RR Lyr stars, and have similar periods but lower luminosities than the short period Cepheids originally found in the Small Magellanic Cloud by Smith et al. (1992). The P/L distribution shows that there is no clear gap between RR Lyr stars and LL Cepheids: the latter seem to fill up the region between the RR Lyr stars and the Classical Cepheids in the classical instability strip. A similar continuity in the classical instability strip has been found in the dI galaxy Phoenix by Gallart et al. (these proceedings). The nature of LL Cepheids is still unclear. Because of their distribution in the $\mathrm{P} / \mathrm{L}$ diagram, they could be related both to the short period Cepheids found in other dI galaxies (IC 1613: 
Dolphin et al. 2001; Leo A: Dolphin et al. 2002; Sextans A: Dolphin et al. 2003; SMC: Sharpee et al. 2002) and to the Anomalous Cepheids found in the dwarf spheroidal galaxies (e.g., Pritzl et al. 2002 and references therein).

The mean apparent magnitude of our RR Lyr stars is $V=24.56 \pm 0.18$ (the error is the standard deviation of the average) while the mean apparent magnitude of the subsample of variables located inside the classical instability strip ( 6 stars) is $V=24.63 \pm 0.14$, yielding a distance modulus of $(\mathrm{m}-\mathrm{M})_{0}=23.36 \pm 0.17$ (see Clementini et al. 2003 for details). The LL Cepheids in our faint sample are on average $V \sim 0.7 \mathrm{mag}$ brighter than the RR Lyr stars. Their period distribution peaks at $P \sim 0.6 \mathrm{~d}$. The lack of LL Cepheids with longer periods could be due to selection effects, since our observations were optimized to provide good coverage of the light curves for variable stars with periods between 0.2 and $0.7 \mathrm{~d}$. We expect to find variables with longer periods in the brighter sample we are currently investigating.

\section{References}

Alard, C. 2000, A\&AS, 144, 363

Baldacci, L., Clementini, G., Held, E.V., Rizzi, L. 2002 in the Workshop Variability with wide field imagers, astro-ph/0303094

Clement, C.M., Rowe, J. 2000, AJ, 120, 2579

Clementini, G., Di Tomaso, S., Di Fabrizio, L., Bragaglia, A., Merighi, R., Tosi, M., Carretta, E., Gratton, R.G., Ivans, I.I., Kinard, A., Marconi, M., Smith, H.A., Wilhelm, R., Woodruff, T., Sneden, C. 2000, AJ, 120, 2054

Clementini, G., Held, E.V., Baldacci, L., Rizzi, L. 2003, AJ, 588, L85

Dolphin, A.E., Saha, A., Skillman, E.D., Tolstoy, E., Cole, A.A., Dohm-Palmer, R.C., Gallagher, J.S., Mateo, M., Hoessel, J.G. 2001, ApJ, 550, 554

Dolphin, A.E., Saha, A., Claver, J., Skillman, E.D., Cole, A.A., Gallagher, J.S., Tolstoy, E., Dohm-Palmer, R.C., Mateo, M. 2002, AJ, 123, 3154

Dolphin, A.E., Saha, A., Skillman, E.D., Dohm-Palmer, R.C., Tolstoy, E., Cole, A.A., Gallagher, J.S., Hoessel, J.G., Mateo, M. 2003, AJ, 125, 1261

Oosterhoff, P.T. 1939, Obs, 64, 140

Pritzl, B.J., Armandroff, T.E., Jacoby, G.H., Da Costa, G.S. 2002, AJ, 124, 1464

Sharpee, B., Stark, M., Pritzl, B.J., Smith, H.A., Silbermann, N.A., Wilhelm, R., Walker, A. 2002, AJ, 123, 3216

Smith, H.A., Silbermann, N.A., Baird, S.R., Graham, J.A. 1992, AJ, 104, 1430

Stetson, P.B. 1994, PASP, 106, 250 